\title{
Mass-varying neutrino in light of cosmic microwave background and weak lensing (Research Note)
}

\author{
G. La Vacca ${ }^{1}$ and D. F. Mota ${ }^{2}$
}

\author{
1 Physics Department "G. Occhialini”, Milano-Bicocca University and I.N.F.N., 20126 Milano, Italy \\ e-mail: lavacca@mib.infn.it \\ 2 Institute of Theoretical Astrophysics, University of Oslo, 0315 Oslo, Norway \\ Received 20 December 2012 / Accepted 23 September 2013
}

\section{ABSTRACT}

\begin{abstract}
Aims. We aim to constrain mass-varying neutrino models using large scale structure observations and produce forecast for the Euclid survey.

Methods. We investigate two models with different scalar field potential and both positive and negative coupling parameters $\beta$. These parameters correspond to growing or decreasing neutrino mass, respectively. We explore couplings up to $|\beta| \lesssim 5$.

Results. In the case of the exponential potential, we find an upper limit on $\Omega_{v} h^{2}<0.004$ at $2 \sigma$ level. In the case of the inverse power law potential the null coupling can be excluded with more than $2 \sigma$ significance; the limits on the coupling are $\beta>3$ for the growing neutrino mass and $\beta<-1.5$ for the decreasing mass case. This is a clear sign for a preference of higher couplings. When including a prior on the present neutrino mass the upper limit on the coupling becomes $|\beta|<3$ at $2 \sigma$ level for the exponential potential. Finally, we present a Fisher forecast using the tomographic weak lensing from an Euclid-like experiment and we also consider the combination with the cosmic microwave background (CMB) temperature and polarisation spectra from a Planck-like mission. If considered alone, lensing data is more efficient in constraining $\Omega_{v}$ with respect to CMB data alone. There is, however, a strong degeneracy in the $\beta-\Omega_{v} h^{2}$ plane. When the two data sets are combined, the latter degeneracy remains, but the errors are reduced by a factor $\sim 2$ for both parameters.
\end{abstract}

Key words. cosmology: theory - dark energy - neutrinos

\section{Introduction}

We study models in which the dark energy (DE) field is coupled to neutrinos (Bjaelde et al. 2008; Brookfield et al. 2006b,a; Amendola et al. 2008; Pettorino et al. 2009). Several authors have shown that important cosmological effects can appear within this class of models, especially when the neutrino mass is sufficiently big for neutrinos to be non-relativistic (Wintergerst et al. 2010; Mota et al. 2008; Pettorino et al. 2010). In this work, we further investigate the cosmological signatures of the massvarying neutrino (MaVaN) models and put constraints on them using the most updated observational data from the cosmic microwave background $(\mathrm{CMB})$ radiation temperature anisotropies spectra, from the large scale structure (LSS), and the supernovae type Ia (SNIa) luminosity distance. We start by investigating two different scalar field potentials and both positive and negative coupling parameters. These parameters corresponds to growing or decreasing neutrino mass, respectively. Finally, we present a Fisher forecast using the tomographic weak lensing from an Euclid-like experiment (Laureijs et al. 2011) in the last section. This is also shown in combination with the CMB temperature and polarisation spectra from a Planck-like mission (Planck Collaboration 2006).

\section{The cosmological background evolution}

The MaVaN models involve a coupling between the DE scalar field and massive neutrinos. The coupling of DE to the neutrinos results in the neutrino mass becoming a function of the scalar field,

$m_{v} \equiv \bar{m}_{v} \mathrm{e}^{\beta \phi}$

where $\bar{m}_{v}$ is a constant. An additional attractive force between neutrinos of strength $2 \beta^{2}$ is mediated by the scalar field exchange. The DE and mass varying neutrinos obey the coupled conservation equations:

$\rho_{\phi}^{\prime}=-3 \mathcal{H}\left(1+w_{\phi}\right) \rho_{\phi}+\beta \phi^{\prime}\left(1-3 w_{v}\right) \rho_{v}$

$\rho_{v}^{\prime}=-3 \mathcal{H}\left(1+w_{v}\right) \rho_{v}-\beta \phi^{\prime}\left(1-3 w_{v}\right) \rho_{v}$

where the energy density and pressure stored in the neutrinos is given by

$\rho_{v}=\frac{1}{a^{4}} \int q^{2} \mathrm{~d} q \mathrm{~d} \Omega \epsilon f_{0}(q), \quad p_{v}=\frac{1}{3 a^{4}} \int q^{2} \mathrm{~d} q \mathrm{~d} \Omega f_{0}(q) \frac{q^{2}}{\epsilon}$.

Here $f_{0}(q)$ is the usual unperturbed background neutrino Fermi-Dirac distribution function:

$f_{0}(\epsilon)=\frac{g_{\mathrm{s}}}{h_{\mathrm{P}}^{3}} \frac{1}{\mathrm{e}^{\epsilon / k_{\mathrm{B}} T_{0}}+1}$,

and $\epsilon^{2}=q^{2}+m_{v}^{2}(\phi) a^{2}$ ( $q$ denotes the comoving momentum). As usual, $g_{\mathrm{s}}, h_{\mathrm{P}}$, and $k_{\mathrm{B}}$ stand for the number of spin degrees of freedom, Planck constant, and Boltzmann constant, respectively.

Taking the energy conservation of the coupled neutrinodark energy system into account, one can immediately find that 
the evolution of the scalar field is described by a modified Klein-Gordon equation:

$\phi^{\prime \prime}+2 \mathcal{H} \phi^{\prime}+a^{2} \frac{\mathrm{d} V}{\mathrm{~d} \phi}=a^{2} \beta\left(\rho_{v}-3 p_{v}\right)$.

Here we investigate two different expressions for the DE potentials, an exponential potential (Wetterich 1988) and an inverse power-law (Binetruy 1999):

$$
V(\phi) \propto \mathrm{e}^{-\sigma \phi}, \quad V(\phi) \propto(M / \phi)^{2} .
$$

The initial conditions for the field $\phi$ and its time derivative are chosen in a recursive way to reproduce the correct value of the assigned density parameters at $a=1$. In the very early universe, neutrinos are still relativistic and almost massless, where $p_{v}=\rho_{v} / 3$ and the coupling term in Eqs. (2), (3), (6) vanishes. However, the coupling term $\sim \beta \rho_{v}$ becomes significantly different from zero as soon as neutrinos become nonrelativistic, affecting the evolution of the field $\phi$. Neutrinos and $\phi$ densities change behaviour for $z<6$ : the value of the scalar field stays almost constant, and the frozen scalar field potential mimics a cosmological constant. According to this model, neutrinos at the present time are still subdominant with respect to cold dark matter (CDM), though they will take the lead in the future. For our choice of the parameters, neutrino pressure terms may be safely neglected for redshifts $z_{\mathrm{nr}}<4$; before that, redshift neutrinos free stream as usual relativistic particles.

\subsection{Perturbed equations}

The perturbed Klein-Gordon equation is given by (Brookfield et al. 2006b):

$$
\begin{aligned}
\ddot{\delta \phi} & +2 H \dot{\delta \phi}+\left(k^{2}+a^{2} \frac{\mathrm{d}^{2} V}{\mathrm{~d} \phi^{2}}\right) \delta \phi+\frac{1}{2} \dot{h} \dot{\phi}= \\
& -a^{2}\left[\frac{\mathrm{d} \ln m_{v}}{\mathrm{~d} \phi}\left(\delta \rho_{v}-3 \delta p_{v}\right)+\frac{\mathrm{d}^{2} \ln m_{v}}{\mathrm{~d} \phi^{2}} \delta \phi\left(\rho_{v}-3 p_{v}\right)\right] .
\end{aligned}
$$

For the neutrinos, we use the perturbed component of the energy momentum conservation equation for the coupled neutrinos $\delta_{v} \equiv \frac{\delta \rho_{v}}{\rho_{v}}$, whilst taking $\gamma=i$ (spatial index) yields the velocity perturbation equation $\theta_{v} \equiv i k_{i} v_{v}^{i}$ with the coordinate velocity $v_{v}^{i} \equiv \mathrm{d} x^{i} / \mathrm{d} \tau$ :

$$
\begin{aligned}
\dot{\delta}_{v}= & 3(H+\beta \dot{\phi})\left(w_{v}-\frac{\delta p_{v}}{\delta \rho_{v}}\right) \delta_{v}-\left(1+w_{v}\right)\left(\theta_{v}+\frac{\dot{h}}{2}\right) \\
& +\beta\left(1-3 w_{v}\right) \dot{\delta \phi}+\frac{\mathrm{d} \beta}{\mathrm{d} \phi} \dot{\phi} \delta \phi\left(1-3 w_{v}\right), \\
\dot{\theta}_{v}= & -H\left(1-3 w_{v}\right) \theta_{v}-\frac{\dot{w}_{v}}{1+w_{v}} \theta_{v}+\frac{\delta p_{v} / \delta \rho_{v}}{1+w_{v}} k^{2} \delta_{v} \\
& +\beta \frac{1-3 w_{v}}{1+w_{v}} k^{2} \delta \phi-\beta\left(1-3 w_{v}\right) \dot{\phi} \theta_{v}-k^{2} \sigma_{v} .
\end{aligned}
$$

\section{Methods and data}

In this paper, we use two methods to test MaVaN theory against cosmological data: the likelihood analysis through Markov-chain Monte-Carlo (MCMC) technique and the Fisher information matrix.

The CMB anisotropy and matter power spectra are calculated by suitably modifying CAMB (Lewis et al. 2000) to include MaVaN's equations as described above. To ensure the accuracy of our calculations, we directly integrate the neutrino distribution function, rather than using the standard velocity weighted series approximation scheme.

For the MCMC analysis, we use a modified version of the publicly available codes CosmoMC (Lewis \& Bridle 2002) to explore the parameter space. We consider the following basic set of parameters:

$\left\{\omega_{\mathrm{b}}, \omega_{\mathrm{CDM}}, H_{0}, z_{\mathrm{re}}, \mathrm{n}_{\mathrm{s}}, \ln \mathrm{A}_{\mathrm{s}}, \omega_{\nu}, \beta\right\}$

and also $\sigma$ when the exponential potential is involved. Here, $\omega_{\mathrm{b}}, \omega_{\mathrm{CDM}}, \omega_{v}$ are the physical baryon, cold dark matter, and neutrino density parameters. Here, $\omega_{i}=\Omega_{i} h^{2}$, where $i=b, C D M, v$, and $h$ is the dimensionless Hubble parameter $H_{0} ; z_{\text {re }}$ is the redshift of reionization; $n_{\mathrm{s}}$ is the scalar spectral index; $A_{\mathrm{s}}$ denotes the amplitude of the scalar fluctuations at a scale of $k=0.05 \mathrm{Mpc}^{-1}$. The sum of the $v$ masses is directly related to the neutrino density parameter through the relation $M_{v}=\Sigma m_{v}=\omega_{v} \cdot 93.5 \mathrm{eV}$, assuming three equal mass $v$ values.

All parameters are given flat priors, unless otherwise stated explicitly. We separately consider positive and negative $\beta$ values in Eq. (1), which correspond to either a growing or decreasing neutrino mass, respectively. We choose to span only the low coupling regime up to $|\beta|<5$, since linear perturbations become unstable for high couplings, e.g. $|\beta| \sim 50$ (Mota et al. 2008; Wintergerst et al. 2010). In our MCMC analysis, we assume that the Universe is spatially flat.

With the aim of obtaining the best estimate of the cosmological parameters, we combine different CMB data sets (WMAP (Komatsu et al. 2011), CBI (Sievers et al. 2007), ACBAR (Reichardt et al. 2009), VSA (Dickinson et al. 2004)) with data from LSS (Tegmark et al. 2006) and SNIa (Amanullah et al. 2010). We also apply additional priors on the Hubble parameter of $H_{0}=74.2 \pm 3.6$ (Riess et al. 2009).

In addition to the likelihood analysis, we test the power of future CMB and weak-lensing data by constraining MaVaN's parameters using the Fisher formalism (Fisher 1935; Tegmark et al. 1997).

\section{MCMC results}

In the case of the exponential potential (7), the plots for $\omega_{v}, \sigma$, and $\beta$ are shown in Fig. 1. For both a growing and decreasing mass, no stringent constraints can be put on the coupling $\beta$, whose upper limit exceeds $|\beta|=5$ at $68 \%$ confidence level (CL). In contrast, the $v$ density parameter is well constrained at $95 \%$ CL: $\omega_{v}<0.0034\left(\omega_{v}<0.0032\right)$ for a decreasing (growing) mass. These limits are $\sim 3$ times narrower than those found in Brookfield et al. (2006b). In terms of current neutrino mass these values result in an upper limit to $m_{v} \simeq 0.32 \mathrm{eV}$. We note here that the results show a common behaviour in the $\beta$ vs. $\omega_{v}$ plots, which are almost symmetric with respect to the sign of $\beta$. A degeneracy appears between the two parameters, so that a high $|\beta|$ agrees with low neutrino content, or, equivalently, low neutrino mass.

In Fig. 2, we reported likelihood contours for the case of the inverse power-law potential. This potential type was not previously considered in Brookfield et al. (2006b). In the $\beta$ vs. $\omega_{\nu}$, plots the zero coupling is apparently excluded with statistical significance that is higher than $95 \%$ confidence level (CL), with $\beta>3$ for the growing neutrino mass and $\beta<-1.5$ for the decreasing mass case at $2 \sigma$. In particular, the growing neutrino mass case exhibits an explicit preference for high $\beta$ values, which agree with Amendola et al. (2008). For $\omega_{v}$, only upper limits can be found at 95\% CL: $\omega_{v}<0.0017\left(\omega_{v}<0.0423\right)$ for decreasing (growing) mass. 

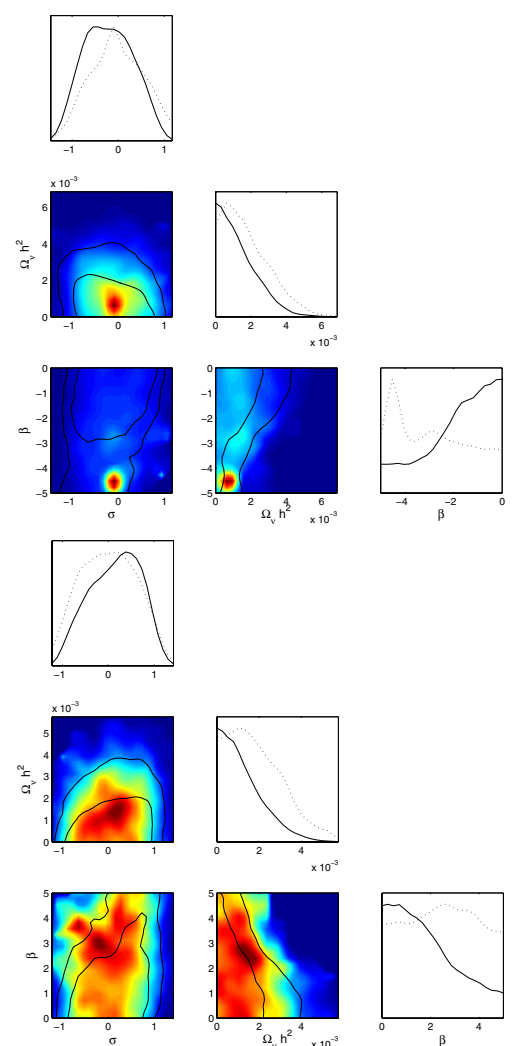

Fig. 1. In the upper (lower) panel, likelihood contours and distributions are shown for decreasing (growing) neutrino mass in the exponential potential case. For the plots on the diagonal, dotted (solid) lines are mean (marginalized) likelihoods of samples. Similarly, black lines on the plots exhibit $1-$ and $2 \sigma$ contours of the marginalised probability distribution, while the colours refer to the mean likelihood degradation from the top (dark red) to lower values.
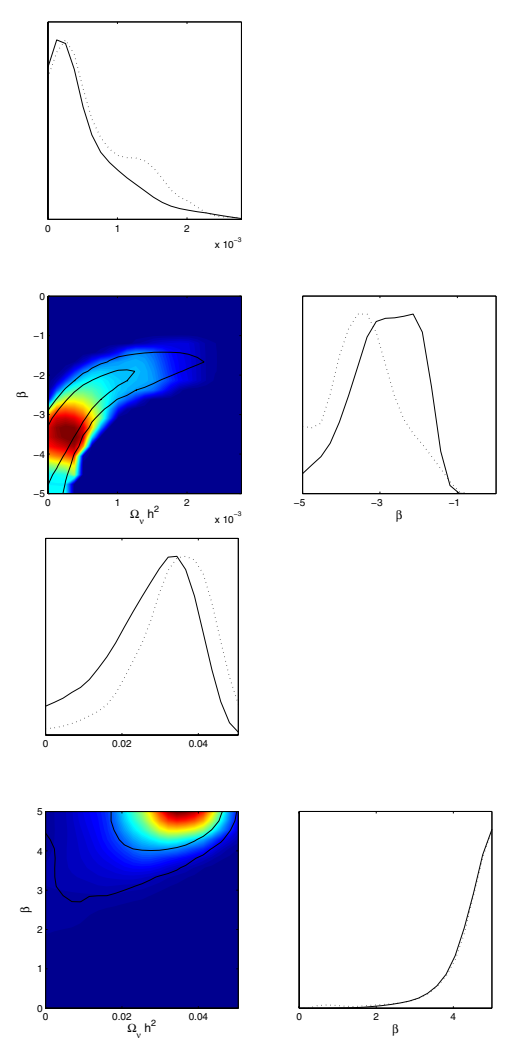

Fig. 2. As in Fig. 1, when the power-law potential is considered.
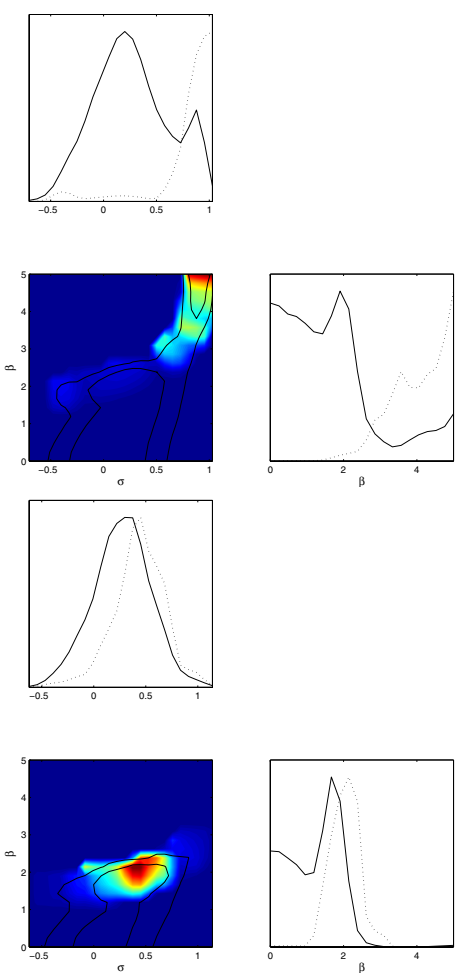

Fig. 3. Upper (lower) panel shows posterior constraints for the exponential potential with growing $v$ mass. Here we fixed $m_{v}=0.2 \mathrm{eV}$ $\left(m_{v}=0.3 \mathrm{eV}\right), \omega_{\mathrm{b}}=0.022, H_{0}=72$.

To exploit complementary information from external measurements, we performed a specific analysis, by fixing the current neutrino mass value using two possible options, $m_{v}=0.2 \mathrm{eV}$ and $m_{v}=0.3 \mathrm{eV}$. These values are within the range of the claimed $v_{\mathrm{e}}$ mass detection in the Heidelberg-Moscow experiment (Klapdor-Kleingrothaus et al. 2004; Klapdor-Kleingrothaus 2005). The KATRIN experiment (Sturm 2011) is also expected to constrain the value of the neutrino mass with a sensitivity in the sub-eV range. Besides fixing $\omega_{v}$, we chose to fix $\Omega_{\mathrm{b}} h^{2}=0.022$, as constraints on this parameter are not significantly modified by neutrino-DE couplings, and $H_{0}=72 \mathrm{~km} \mathrm{~s}^{-1} \mathrm{Mpc}^{-1}$.

As shown in Figs. 3 and 4, the most significant result is the improvement in the limits on the coupling $|\beta|$, which has an upper value of the order between 2 or 3 , and the distributions for $\sigma$ become narrower, but are always compatible with zero at $68 \%$ CL. The complex structure for the likelihood contours in the upper panel of Fig. 3 can be mainly ascribed to the peculiar features of the likelihood in Fig. 1, which are completely lost when considering a prior with higher mass.

\section{Fisher matrix forecasts}

In this section, we show Fisher matrix results from the combination of the CMB anisotropies and the tomographic weak lensing (TWL) spectra. We considered the exponential potential with an exponential coupling. The fiducial parameters $\theta_{\alpha}$ are shown in Table 1. These two sets correspond to the maximum area of the likelihood as determined above and are chosen to be exactly the same, except for the sign of $\beta$.

The Fisher matrix for CMB power spectrum (Zaldarriaga \& Seljak 1997; Zaldarriaga et al. 1997; Rassat et al. 2008) is calculated using Planck mission (Planck Collaboration 2006) specifications. We assume that we only use the $143 \mathrm{GHz}$ channel as 

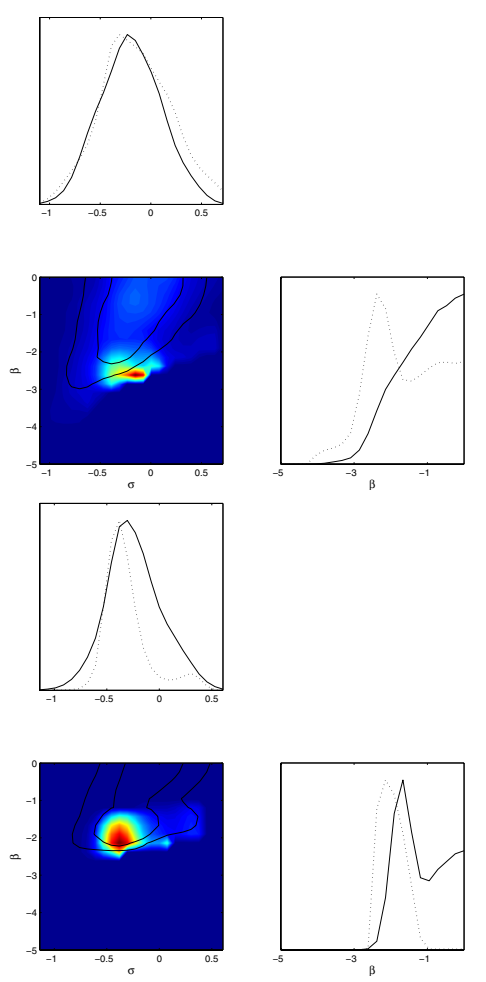

Fig. 4. Upper (lower) panel shows posterior constraints for the exponential potential with decreasing $v$ mass. Here we fixed $m_{v}=0.2 \mathrm{eV}$ $\left(m_{v}=0.3 \mathrm{eV}\right), \omega_{\mathrm{b}}=0.022, H_{0}=72$.

Table 1. Fiducial cosmological parameters for the exponential potential, which are consistent with Fig. 1.

\begin{tabular}{lcccccc}
\hline \hline Parameter & $\omega_{\mathrm{b}}$ & $\omega_{\mathrm{CDM}}$ & $H_{0}$ & $z_{\text {re }}$ & $n_{\mathrm{s}}$ & $\ln A_{\mathrm{s}}$ \\
Value & 0.0224 & 0.1097 & 69 & 11 & 0.95 & $2.09 \times 10^{-9}$ \\
\hline Parameter & $\omega_{v}$ & $\sigma$ & $\beta$ & & & \\
Value & 0.0010 & 0.02 & \pm 1.9 & & & \\
\hline
\end{tabular}

the science channel. This channel has a beam of $\theta_{\mathrm{fwhm}}=7.1^{\prime}$ and sensitivities of $\sigma_{\mathrm{T}}=2.2 \mu K / K$ and $\sigma_{\mathrm{P}}=4.2 \mu K / K$. To account for the galactic plane cut, we take $f_{\text {sky }}=0.80$. Note that we use $\ell_{\text {min }}=30$ as a minimum $\ell$-mode to avoid problems with polarization foregrounds and subtleties when modelling of the integrated Sachs-Wolfe effect.

The Fisher matrix for TWL (Hu \& Jain 2004) is calculated using the forthcoming Euclid mission ${ }^{1}$ specifications (Laureijs et al. 2011). The survey area covered by the experiment is $15000 \mathrm{deg}^{2}$, while the density is 30 galaxies per $\operatorname{arcmin}^{2}$. The distribution of the galaxy number on the redshift and solid angle is $n(z)=n_{0} z^{2} \mathrm{e}^{-\left(z / z_{0}\right)^{1.5}}$ with a median redshift $\bar{z}=0.9$. The photometric redshift error is $0.05(1+z)$. We consider the five bin case as reference case. Non-linear corrections of the matter power spectra are calculated using HALOFIT (Smith et al. 2003). Since this procedure is suitably fitted to $\Lambda \mathrm{CDM} N$-body simulations, it can lead to errors of the order of $20 \%$ on Fisher outputs, if used for models different from $\Lambda$ CDM (see e.g. Casarini et al. 2011). However, we use HALOFIT in the absence of suitable extensions for $\mathrm{MaVaN}$, and report results up to $\ell_{\max }=1000$ in the mildly non-linear regime. This conservative choice also prevents us from the effects of baryons on the matter power spectra and weak-lensing spectra (Casarini et al. 2012).

As shown in Table 2, TWL is able to put constraints stronger than $\mathrm{CMB}$ on $\beta<0$ and $\omega_{v}$, with an improvement of $\sim 10 \%$

\footnotetext{
1 http://www. euclid-ec.org
}

Table 2. 1- $\sigma$ error estimations of cosmological parameters for an exponential potential with $\beta<0$, using Planck-like CMB and Euclid-like tomographic weak lensing (TWL) data.

\begin{tabular}{lccc}
\hline \hline Dataset & $\sigma_{\omega_{\nu}}$ & $\sigma_{\sigma}$ & $\sigma_{\beta}$ \\
\hline TWL & 0.00043 & 0.024 & 1.17 \\
CMB & 0.00064 & 0.014 & 1.28 \\
TWL+CMB & 0.00025 & 0.012 & 0.69 \\
\hline
\end{tabular}

Table 3. Same as Table 2 but with $\beta>0$.

\begin{tabular}{lccc}
\hline \hline Dataset & $\sigma_{\omega_{\nu}}$ & $\sigma_{\sigma}$ & $\sigma_{\beta}$ \\
\hline TWL & 0.00045 & 0.028 & 2.04 \\
CMB & 0.00054 & 0.019 & 1.71 \\
TWL+CMB & 0.00026 & 0.012 & 1.15 \\
\hline
\end{tabular}
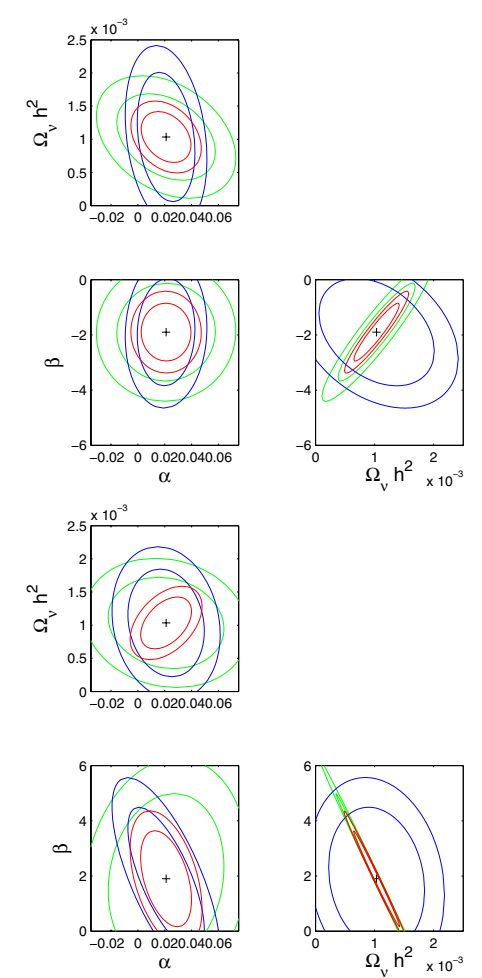

Fig. 5. Upper (lower) panel shows $68 \%$ and $95 \%$ likelihood confidence levels for the exponential potential, in the decreasing (growing) mass case. The green lines are obtained considering an Euclid-like TWL survey. The blue lines are for a Planck-like CMB mission. The red lines are for the combination of the two.

and $>30 \%$, respectively. Instead CMB is more efficient in constraining $\sigma$, gaining $\sim 50 \%$ over TWL. The combination of the two observables can improve constraints on all parameters. Analogous comments can be made for the $\beta>0$ in Table 3 with the only difference that CMB can constrain $\beta$ better than TWL.

In Fig. 5, we show $1 \sigma$ and $2 \sigma$ likelihood contours for $\omega_{\nu}, \beta$ and $\sigma$, after marginalising over the other parameters. It is clear that neither Planck nor Euclid alone can be able to constrain a non-zero coupling $\beta \sim O(1)$; only TWL is slightly more efficient than CMB in the $\beta<0$ case. In this case the combination of the two can exclude a null coupling with a significance higher than $2 \sigma$. We note that higher multipoles can significantly reduce the limits on $\beta$ and the other parameters. If we suppose, for instance, that HALOFIT is appropriate for our models at multipoles up to $\ell_{\max }=5000$ in the TWL Fisher matrix (see Fig. 6), the estimated errors are then clearly reduced with a factor $\sim 2$ with 

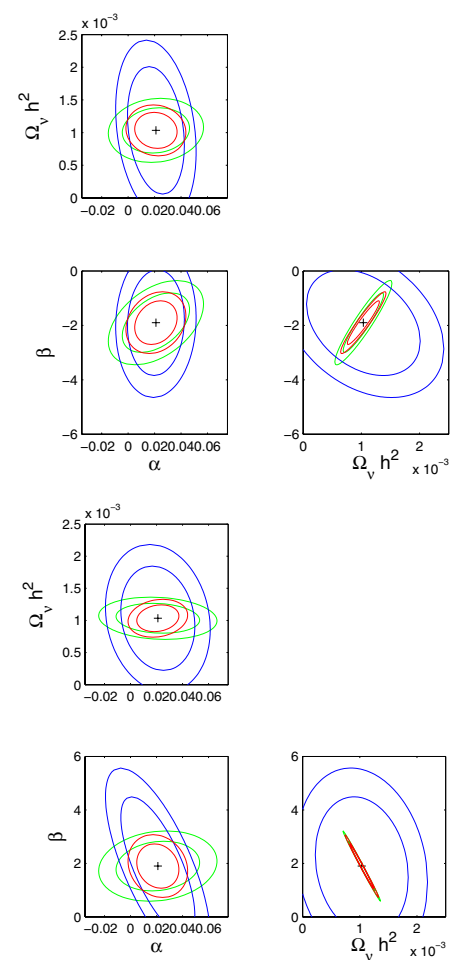

Fig. 6. As in Fig. 5 with 5 bins and $\ell_{\max }=5000$.

respect to Fig. 6. In this case, Euclid would be able to exclude the zero value both for $\beta$ and $\omega_{v}$ with high statistical significance.

Despite this limitation, a very interesting result is that TWL, whether alone or in combination with $\mathrm{CMB}$, is able to find a lower value of $\Omega_{\gamma} h^{2}$ with an error that is half of the CMB error if taken alone. Moreover, a strong degeneracy can be found in the $\omega_{v}-\beta$ plane with regard to the TWL ellipses and also in combination with CMB. This effect appears in both cases in a symmetric way, showing that lower $|\beta|$ values allow higher neutrino mass. The $\Omega_{v} h^{2}-\beta$ degeneracy could be eventually broken from external priors on $m_{v}$. On the other hand, no noteworthy remark can be said about the $\sigma$ parameter, whose constrain remains compatible with zero for the combination of the two observables.

\section{Discussion and conclusions}

In this work, we focused on the hypothesis that the origin of cosmic acceleration can be attributed to a quintessence scalar field coupled to massive neutrinos. First, we updated and extended parameter constraints using the most recent available data from SNIa, CMB, and LSS observations. We considered both an exponential and a power law potential with growing $(\beta>0)$ or decreasing $(\beta<0)$ neutrino mass.

The cosmological data did not place strong constraints on the coupling parameter in the low coupling range or for the neutrino density parameter, on which only upper limits can be placed in any of these cases. The main outcome of the analysis was that $\beta$ values $\sim O(1)$ are compatible with actual data with a neutrino mass $m_{v} \lesssim 0.32 \mathrm{eV}$.

Therefore we do have not enough information at the moment to exclude a possible coupling between the neutrino and DE field in either the low or the high coupling regime. New and precise data from observables related to the recent evolution of the universe are necessary with a deeper understanding of the MaVaN theory dynamics. In this sense, the forthcoming Euclid weak-lensing and galaxy clustering data represent the turning point for the future progress in cosmology.
With this aim, a Fisher matrix study was accomplished by considering future data release from missions, such as Planck for CMB spectra and Euclid for tomographic weak lensing spectra. The latter experiment is more efficient in improving present constraints on $\omega_{v}$, even considering a cautious use of multipoles up to $\ell_{\max }=1000$. This choice prevents us from including highly non-linear features, which are not correctly predicted by HALOFIT for MaVaN theories. Combining Euclid data with complementary information from Planck reduces the estimated error to about a factor 2 with respect to considering the two datasets alone. It is worth to mention that the strong degeneracy between $\beta$ and $\omega_{v}$ could eventually be broken by an external prior on the neutrino mass.

A crucial point for the forthcoming Euclid mission is to study and implement an efficient way of including non-linear corrections on the matter power spectrum calculations with HALOFIT being only suitable for $\Lambda \mathrm{CDM}$ cosmologies. Delving into the deep non-linear regime of matter perturbations could significantly improve parameter estimation by fully exploiting the high potentiality of the TWL from Euclid.

Acknowledgements. D.F.M. thanks the Research Council of Norway.

\section{References}

Amanullah, R., Lidman, C., Rubin, D., et al. 2010, ApJ, 716, 712

Amendola, L., Baldi, M., \& Wetterich, C. 2008, Phys. Rev. D, 78, 023015 Binetruy, P. 1999, Phys. Rev. D, 60, 063502

Bjaelde, O. E., Brookfield, A. W., van de Bruck, C., et al. 2008, JCAP, 0801, 026 Brookfield, A., van de Bruck, C., Mota, D., \& Tocchini-Valentini, D. 2006a, Phys. Rev. Lett., 96, 061301

Brookfield, A. W., van de Bruck, C., Mota, D., \& Tocchini-Valentini, D. 2006b, Phys. Rev. D, 73, 083515

Casarini, L., La Vacca, G., Amendola, L., Bonometto, S. A., \& Maccio, A. V. 2011, JCAP, 1103, 026

Casarini, L., Bonometto, S. A., Borgani, S., et al. 2012, A\&A, 542, A126

Dickinson, C., Battye, R. A., Carreira, P., et al. 2004, MNRAS, 353, 732

Fisher, R. A. 1935, J. Roy. Statist. Soc., 98, 39

Hu, W., \& Jain, B. 2004, Phys. Rev. D, 70, 043009

Klapdor-Kleingrothaus, H. V. 2005, in Proc. of XI Int. Work. on Neutrino Telescopes, Febr. 22-25, Venice, Italy, ed. M. Baldo-Ceolin, Padova Univ., 215

Klapdor-Kleingrothaus, H., Krivosheina, I., Dietz, A., \& Chkvorets, O. 2004, Phys. Lett. B, 586, 198

Komatsu, E., Smith, K. M., Dunkley, J., et al. 2011, ApJS, 192, 18

Laureijs, R., Amiaux, J., Arduini, S., et al. 2011 [arXiv: 1110. 3193]

Lewis, A., \& Bridle, S. 2002, Phys. Rev. D, 66, 103511

Lewis, A., Challinor, A., \& Lasenby, A. 2000, ApJ, 538, 473

Mota, D., Pettorino, V., Robbers, G., \& Wetterich, C. 2008, Phys. Lett. B, 663, 160

Pettorino, V., Mota, D. F., Robbers, G., \& Wetterich, C. 2009, AIP Conf. Proc., 1115, 291

Pettorino, V., Wintergerst, N., Amendola, L., \& Wetterich, C. 2010, Phys. Rev. D, 82,123001

Planck Collaboration 2006 [arXiv: astro-ph/0604069]

Rassat, A., Amara, A., Amendola, L., et al. 2008, MNRAS, submitted [arXiv:0810.0003]

Reichardt, C., Ade, P., Bock, J., et al. 2009, ApJ, 694, 1200

Riess, A. G., Macri, L., Casertano, S., et al. 2009, ApJ, 699, 539

Sievers, J. L., Achermann, C., Bond, J., et al. 2007, ApJ, 660, 976

Smith, R. Peacock, J. A., Jenkins, A., et al. 2003, MNRAS, 341, 1311

Sturm, M. 2011 [arXiv: 1111.4773]

Tegmark, M., Taylor, A., \& Heavens, A. 1997, ApJ, 480, 22

Tegmark, M., Eisenstein, D. J., Strauss, M. A., et al. 2006, Phys. Rev. D, 74, 123507

Wetterich, C. 1988, Nucl. Phys. B, 302, 645

Wintergerst, N., Pettorino, V., Mota, D., \& Wetterich, C. 2010, Phys. Rev. D, 81, 063525

Zaldarriaga, M., \& Seljak, U. 1997, Phys. Rev. D, 55, 1830

Zaldarriaga, M., Spergel, D. N., \& Seljak, U. 1997, ApJ, 488, 1 